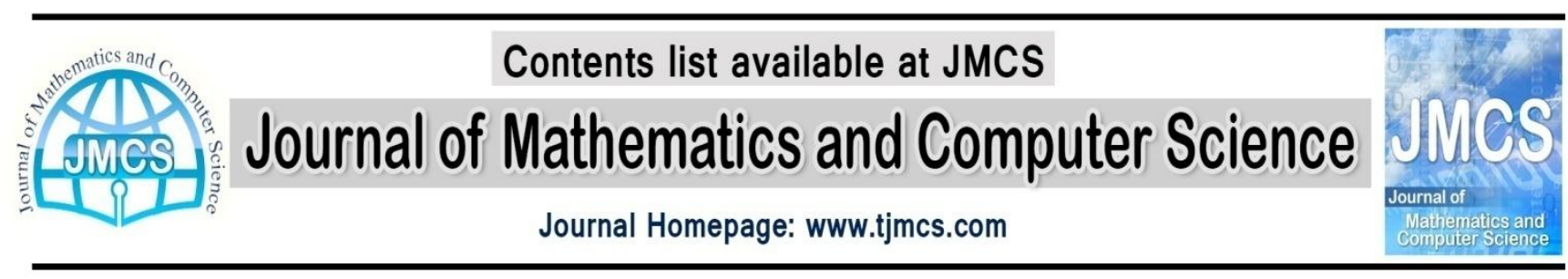

\title{
Numerical solution for Poisson fractional equation via finite differences theta-method
}

\author{
Mohammad Aslefallah ${ }^{1}$, Davood Rostamy ${ }^{2}$ \\ 1,2 Department of Mathematics, Imam Khomeini International University, Qazvin, Iran
}

E-mail: maslefallah@gmail.com,maslefallah@alm.ikiu.ac.ir

Article history:

Received July 2014

Accepted August 2014

Available online August 2014

\begin{abstract}
In this paper we examine $\theta$-method for solving fractional Possion differential equations for $(0 \leq \theta \leq 1)$. Consistency, stability and convergence analysis of the method is discussed. At the end, numerical examples have been presented. The obtained results reveal that the proposed technique is very effective, convenient and quite accurate to such considered problems.
\end{abstract}

Keywords: Fractional PDE (FPDE), Finite differences $\theta$-method, Riemann-Liouville derivative, Shifted Grunwald formula.

Mathematics subject classification (2010): 65M06, 65N12, $26 \mathrm{A33}$.

\section{Introduction}

In the recent years, the theory of fractional calculus has obtained considerable popularity and importance as generalizations of integer-order partial differential evolution equations. Many natural phenomena can be present by fractional differential equations. Many authors in different fields such as chemical physics, fluid flows, electrical networks, viscoelasticity, try to modeling of these phenomena by fractional differential equations. 
Fractional calculus applied to model many meaningful things, such as fractional differential equation can model price volatility in finance ([4],[13]), model fast spreading of pollutants in hydrology ([15]), model the particle motions in a heterogeneous environment and long particle jumps of the anomalous diffusion in physics ([1],[5],[9]). The most common hydrologic and physics application of fractional calculus is the generation of fractional Brownian motion as a representation of aquifer material with long-range correlation structure $([2],[11])$. Other exact description of the applications of engineering, mechanics and mathematics et al., the literature is made to ([6],[12],[16],[19]). Many cases of the real physical processes could be modeled in a reliable manner using fractional-order differential equations ([18]).

Most fractional differential equations do not have exact analytic solutions, so approximation and numerical techniques must be used.

Fractional space derivatives are used to model anomalous diffusion or dispersion, where a particle plume spreads at a rate inconsistent with the classical Brownian motion model. When a fractional derivative replaces the second derivative in a diffusion or dispersion model, it leads to enhanced diffusion. The fractional Poisson equation is often encountered in heat and mass transfer theory, fluid mechanics, elasticity, electrostatics and other areas of mechanics and physics.

In this paper, we develop the basic theory of numerical solution for the fractional poission differential equation

$$
\nabla^{\alpha} u(x, y)=\frac{\partial^{\alpha} u(x, y)}{\partial x^{\alpha}}+\frac{\partial^{\alpha} u(x, y)}{\partial y^{\alpha}}=f(x, y)
$$

on a finite domain $[0,1] \times[0,1]$. Here, we assume that $1 \leq \alpha \leq 2$ as the fractional order of the derivatives. The function $f(x, y)$ is a known function.

Initial conditions are $u(x ; 0)=0, u(0 ; y)=0$ and Dirichlet boundary conditions are as follows:

$$
u(1, y)=g(y) \quad \text { and } \quad u(x, 1)=h(x)
$$

Published papers on the numerical solution of fractional partial differential equations are scarce. The theta-method is generalization of implicit, explicit and Crank-Nicholson methods.

\section{Preliminaries}

For implementation of this method we need to the following definitions.(see [12],[16])

Definition 2.1. (Riemann-Liouville Fractional derivative) If $f$ be a real function and has continues derivatives of integer order $n$, then

$$
D_{x}^{\alpha} f(x)=\frac{d^{n} f(x)}{d x^{n}}=\frac{1}{\Gamma(n-\alpha)} \cdot \frac{d^{n}}{d x^{n}} \int_{0}^{\infty} \frac{f(t)}{(x-t)^{\alpha+1-n}} d t
$$

is Riemann-Liouville fractional derivative of order $\alpha$ that $n-1<\alpha \leq n$. 
Definition 2.2. (Shifted Grunwald formula) For $1<\alpha \leq 2$ shifted Grunwald formula defines:

$$
\frac{d^{n} f}{d x^{n}}=\lim _{M \rightarrow \infty} \frac{1}{h^{\alpha}} \sum_{k=0}^{M} g_{\alpha, k} f(x-(k-1) h),
$$

such that shifted Grunwald estimates for fractional derivative defines :

$$
\frac{d^{n} f}{d x^{n}}=\frac{1}{h^{\alpha}} \sum_{k=0}^{M} g_{\alpha, k} \cdot f(x-(k-1) h)+O\left(h^{\alpha}\right),
$$

And $M$ is a positive integers and $h=\frac{x_{R}-x_{L}}{M}$,

Moreover, normalized Grunwald Weights are defined by:

$$
\mathrm{g}_{\alpha, \mathrm{k}}=(-1)^{\mathrm{k}} \cdot \frac{\Gamma(\alpha+1)}{\Gamma(\mathrm{k}+1) \Gamma(\alpha-\mathrm{k}+1)} \quad, \mathrm{k}=0,1,2, \ldots
$$

Definition 2.3. (Taylor expansion of fractional order) If $f$ be a continuous function that for any positive integer $k$ and any $1<\alpha \leq 2$ has fractional derivative of order $k \alpha$ then fractional Taylor expansion is as follows:

$$
f(x+h)=\sum_{k=0}^{\infty} \frac{h^{k \alpha}}{\Gamma(1+k \alpha)} \cdot f^{(k \alpha)}(x)
$$

such that $f(x+h)=E_{\alpha}\left(h^{\alpha} D_{x}^{\alpha}\right) f(x)$, that $E_{\alpha}(x)=\sum_{k=0}^{\infty} \frac{x^{k}}{\Gamma(1+k \alpha)}$, is Mittag-Leffler function.

\section{Formulation of problem by $\theta$-method}

Here, we assume $\mathrm{h}=\Delta \mathrm{x}$ for $\mathrm{x}$-axis and $\mathrm{k}=\Delta \mathrm{y}$ for $\mathrm{y}$-axis as grid size therefore we have:

$$
\begin{cases}x_{i}=i h, & i=1,2, \ldots, M \\ y_{j}=j k, & j=1,2, \ldots, K\end{cases}
$$

Now, if $U_{i, j}=U\left(x_{i}, t_{j}\right)$ represent the numerical approximation solution with $\theta$ - method, we have:

$$
\theta \delta_{\alpha, x} U_{i, j+1}+(1-\theta) \delta_{\alpha, x} U_{i, j}+\theta^{\prime} \delta_{\alpha, y} U_{i+1, j}+\left(1-\theta^{\prime}\right) \delta_{\alpha, y} U_{i, j}=f_{i, j}
$$

such that we define

$$
\delta_{\alpha, \mathrm{x}} \mathrm{U}_{\mathrm{i}, \mathrm{j}}=\frac{1}{(\Delta \mathrm{x})^{\alpha}} \sum_{s=0}^{\mathrm{i}+1} \mathrm{~g}_{\alpha, \mathrm{s}} \cdot \mathrm{U}_{\mathrm{i}-\mathrm{s}+1, \mathrm{j}} \text { and } \delta_{\alpha, \mathrm{y}} \mathrm{U}_{\mathrm{i}, \mathrm{j}}=\frac{1}{(\Delta \mathrm{y})^{\alpha}} \sum_{\mathrm{t}=0}^{\mathrm{i}+1} \mathrm{~g}_{\alpha, \mathrm{t}} \cdot \mathrm{U}_{\mathrm{i}, \mathrm{j}-\mathrm{t}+1} \text {, }
$$

In other words, we can rewrite equation (3.1) as follows: 


$$
\begin{aligned}
& \frac{1}{h^{\alpha}}\left(\theta \sum_{p=0}^{i+1} g_{\alpha, p} \cdot U_{i-p+1, j+1}+(1-\theta) \sum_{q=0}^{i+1} g_{\alpha, q} \cdot U_{i-q+1, j}\right) \\
& +\frac{1}{k^{\alpha}}\left(\theta^{\prime} \sum_{s=0}^{i+1} g_{\alpha, s} \cdot U_{i+1, j-s+1}+\left(1-\theta^{\prime}\right) \sum_{z=0}^{i+1} g_{\alpha, z} \cdot U_{i, j-z+1}\right)=f_{i, j},
\end{aligned}
$$

In general case for $\left(0<\theta, \theta^{\prime} \leq 1\right)$ from (3.2) by rearraging and simplifying we have:

$$
U_{i+1, j+1}=\frac{1}{E}\left(f_{i, j}-A-B-C-D\right) \text {, }
$$

where:

$$
\begin{array}{cl}
A=\frac{\theta}{h^{\alpha}} \sum_{p=1}^{i+1} g_{\alpha, p} \cdot U_{i-p+1, j+1}, & B=\frac{(1-\theta)}{h^{\alpha}} \sum_{q=0}^{i+1} g_{\alpha, q} \cdot U_{i-q+1, j}, \\
C=\frac{\theta^{\prime}}{k^{\alpha}} \sum_{s=1}^{i+1} g_{\alpha, s} \cdot U_{i+1, j-s+1}, & D=\frac{\left(1-\theta^{\prime}\right)}{k^{\alpha}} \sum_{z=0}^{i+1} g_{\alpha, z} \cdot U_{i, j-z+1}, \\
\text { and } & E=\frac{\theta}{h^{\alpha}}+\frac{\theta^{\prime}}{k^{\alpha},}
\end{array}
$$

In special case, for $\theta=\theta^{\prime}=0, h=k$ and $M=N$ we have:

$$
\frac{1}{h^{\alpha}}\left(\sum_{q=0}^{i+1} g_{\alpha, q} \cdot U_{i-q+1, j}+\sum_{z=0}^{i+1} g_{\alpha, z} \cdot U_{i, j-z+1}\right)=f_{i, j},
$$

\section{Analysis of Stability, Consistency and Convergence}

If $U$ be an approximated solution and $u$ be exact solution and $F_{i, j}(U)=0$ represent approximated difference equation of FPDE at mesh point $\left(x_{i}, y_{j}\right)$. By substitution $U$ with $u$ value $T_{i, j}=F_{i, j}(u)$ represented local truncation error $(\mathrm{LTE})$ at mesh point $\left(\mathrm{x}_{\mathrm{i}}, \mathrm{y}_{\mathrm{j}}\right)$.

Theorem 4.1. LTE for finite differences method (3.3) is:

$$
\mathrm{T}_{\mathrm{i}, \mathrm{j}}=\mathrm{h}^{\alpha}\left(\sum_{\mathrm{s}=0}^{\infty} \mathrm{g}_{\alpha, \mathrm{s}} \cdot \frac{(1-\mathrm{s})^{2 \alpha}}{(2 \alpha) !}\right)\left[\frac{\partial^{2 \alpha} \mathrm{u}}{\partial \mathrm{x}^{2 \alpha}}+\frac{\partial^{2 \alpha} \mathrm{u}}{\partial \mathrm{y}^{2 \alpha}}\right]_{\mathrm{i}, \mathrm{j}}+\mathrm{O}\left(\mathrm{h}^{2 \alpha}\right),
$$


Proof. If $U$ be an approximated solution and $u$ be exact solution and $F_{i, j}(U)=0$ represent approximated difference equation of FPDE at mesh point $\left(x_{i}, y_{j}\right)$. By substitution $U$ with $u$ value $\mathrm{T}_{\mathrm{i}, \mathrm{j}}=\mathrm{F}_{\mathrm{i}, \mathrm{j}}(\mathrm{u})$ represented local truncation error (LTE) at mesh point $\left(\mathrm{x}_{\mathrm{i}}, \mathrm{y}_{\mathrm{j}}\right)$.

From (3.3) we have:

$$
T_{i, j}=\frac{1}{h^{\alpha}}\left(\sum_{q=0}^{i+1} g_{\alpha, q} \cdot U_{i-q+1, j}+\sum_{z=0}^{i+1} g_{\alpha, z} \cdot U_{i, j-z+1}\right)-f_{i, j}
$$

and by the fractional Taylor's expansion:

$$
\begin{aligned}
& T_{i, j}=\frac{1}{h^{\alpha}}\left(\sum_{q=0}^{i+1} g_{\alpha, q} \cdot\left(U_{i, j}+\frac{[(1-q) h]^{\alpha}}{\alpha !}\left(\frac{\partial^{\alpha} U}{\partial x^{\alpha}}\right)_{i, j}+\frac{[(1-q) h]^{2 \alpha}}{(2 \alpha) !}\left(\frac{\partial^{2 \alpha} U}{\partial x^{2 \alpha}}\right)_{i, j}+\cdots\right)\right) \\
& +\frac{1}{h^{\alpha}}\left(\sum_{z=0}^{i+1} g_{\alpha, z} \cdot\left(U_{i, j}+\frac{[(1-z) h]^{\alpha}}{\alpha !}\left(\frac{\partial^{\alpha} U}{\partial y^{\alpha}}\right)_{i, j}+\frac{[(1-z) h]^{2 \alpha}}{(2 \alpha) !}\left(\frac{\partial^{2 \alpha} U}{\partial y^{2 \alpha}}\right)_{i, j}+\cdots\right)\right)-f_{i, j}
\end{aligned}
$$

From (see [8]) :

$$
\sum_{\mathrm{k}=0}^{\infty} \mathrm{g}_{\alpha, \mathrm{k}}=0
$$

and also we can prove that:

$$
\sum_{\mathrm{k}=0}^{\infty} \mathrm{g}_{\alpha, \mathrm{k}}(1-\mathrm{k})^{\alpha}=\alpha !
$$

By considering (4.3) , (4.4) and (4.5 we obtain:

$$
T_{i, j}=\left(\nabla^{\alpha} U-f\right)_{i, j}+h^{\alpha}\left(\sum_{s=0}^{\infty} g_{\alpha, s} \frac{(1-s)^{2 \alpha}}{(2 \alpha) !}\right)\left[\frac{\partial^{2 \alpha} u}{\partial x^{2 \alpha}}+\frac{\partial^{2 \alpha} u}{\partial y^{2 \alpha}}\right]_{i, j}+O\left(h^{2 \alpha}\right)
$$


We know :

$$
\left(\nabla^{\alpha} U-f\right)_{i, j}=0
$$

because $u$ is exact solution of equation (1.1).

Therefore, for LTE we conclude that

$$
\mathrm{T}_{\mathrm{i}, \mathrm{j}}=\mathrm{h}^{\alpha}\left(\sum_{s=0}^{\infty} \mathrm{g}_{\alpha, \mathrm{s}} \cdot \frac{(1-\mathrm{s})^{2 \alpha}}{(2 \alpha) !}\right)\left[\frac{\partial^{2 \alpha} \mathrm{u}}{\partial \mathrm{x}^{2 \alpha}}+\frac{\partial^{2 \alpha} \mathrm{u}}{\partial \mathrm{y}^{2 \alpha}}\right]_{\mathrm{i}, \mathrm{j}}+\mathrm{O}\left(\mathrm{h}^{2 \alpha}\right)
$$

Theorem 4.2. The solution of equation (3.3) exists and is unique.

Proof. (see [3])

Corollary 4.3. Theorem 4.1 shows that this method is consistent, because for $h \rightarrow 0$ and $k \rightarrow 0$, LTE tend to zero.

Corollary 4.4. According to stability analysis and consistency analysis of this method, now from LaxRichtmyer's equivalence theorem this method is convergence.

\section{Numercal Example}

Example 5.1. Consider equation (1.1) on $\mathrm{S}=\{(\mathrm{x}, \mathrm{t}) \mid 0 \leq \mathrm{x} \leq 1,0 \leq \mathrm{t} \leq 1\}$ where:

$$
f(x, y)=\Gamma(\alpha+1)\left(x^{\alpha}+y^{\alpha}\right)
$$

with initial and boundary conditions:

$$
\left\{\begin{array}{cc}
u(x, 0)=0, & u(0, y)=0 \\
u(x, 1)=x^{\alpha}, & u(1, y)=y^{\alpha}
\end{array}\right.
$$

We can show that exact solution is: $u(x, y)=(x y)^{\alpha}$.

The fractional Poisson absolute-error is identified by:

$$
\text { Error }=\frac{1}{(m-1)^{2}} \sqrt{\sum_{i, j=1}^{m-1}\left(U_{i, j}-u_{i, j}\right)^{2}}
$$


The values of errors are shown in Table 1 for the above example problem for different values of $h, \alpha$ and $\theta$. Figures $1-4$ having different values of $h, \alpha$ and $\theta$ verify the efficiency of the proposed scheme.

Table 1: Error for different values of $h, \alpha$ and $\theta$.

\begin{tabular}{|l|l|l|l|l|l|}
\hline & $\mathrm{h}=\mathrm{k}$ & $\theta=0.6$ & $\theta=0.7$ & $\theta=0.8$ & $\theta=0.9$ \\
\hline \multirow{2}{*}{$\alpha=1.25$} & $\mathrm{~h}=0.10$ & 0.005734 & 0.006295 & 0.006818 & 0.007308 \\
\cline { 2 - 6 } & $\mathrm{h}=0.05$ & 0.001558 & 0.001737 & 0.001909 & 0.002074 \\
\hline \multirow{2}{*}{$\alpha=1.50$} & $\mathrm{~h}=0.10$ & 0.005094 & 0.005676 & 0.006193 & 0.006663 \\
\cline { 2 - 6 } & $\mathrm{h}=0.05$ & 0.001516 & 0.001589 & 0.001802 & 0.001984 \\
\hline \multirow{2}{*}{$\alpha=1.75$} & $\mathrm{~h}=0.10$ & 0.004715 & 0.005788 & 0.005298 & 0.006223 \\
\cline { 2 - 6 } & $\mathrm{h}=0.05$ & 0.001322 & 0.002498 & 0.002717 & 0.002414 \\
\hline
\end{tabular}

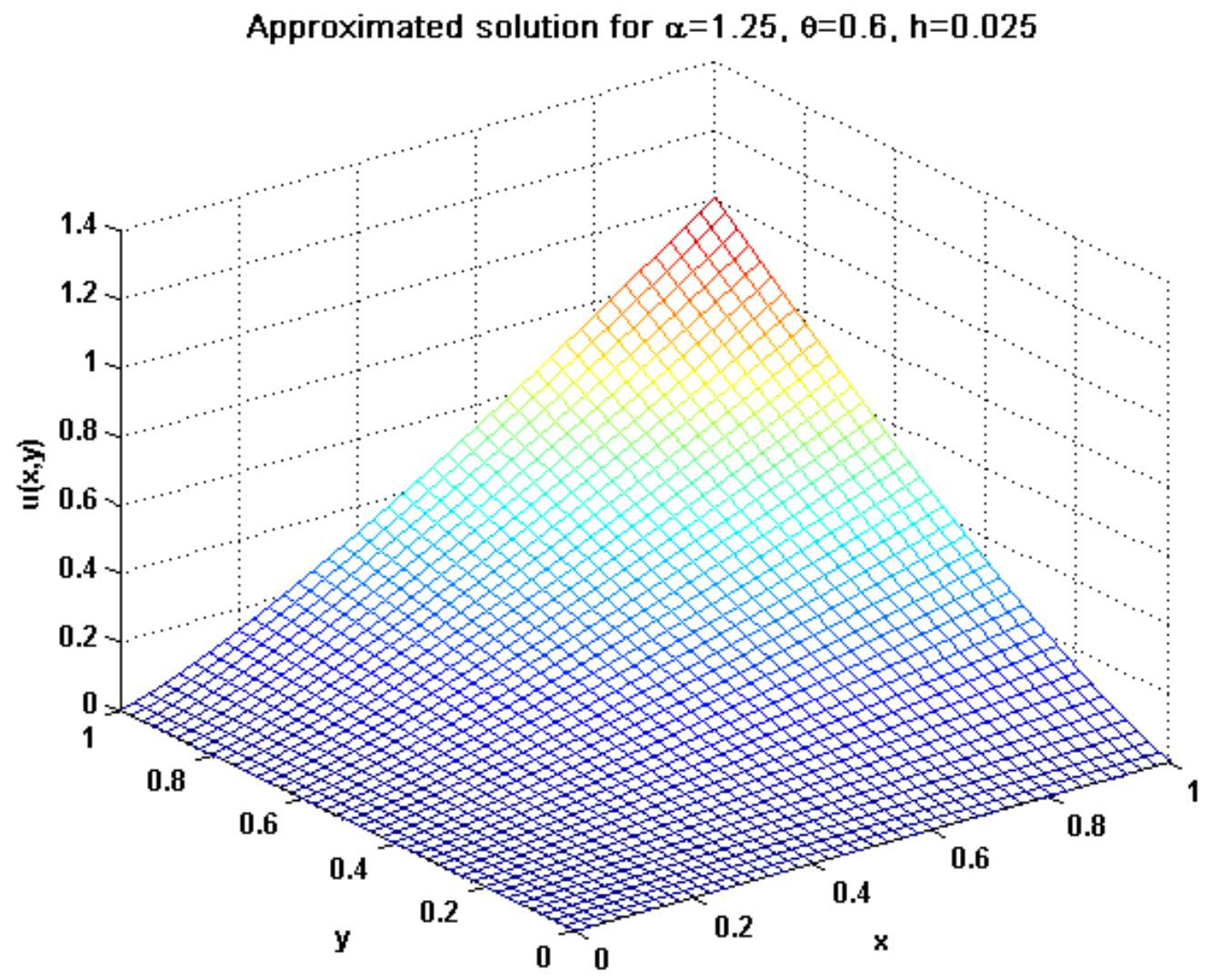

Figure 1: 3D meshed surface plot of numerical solutions for the Example 5.1 with $\alpha=1.25, \theta=0.6, h=0.025$ 


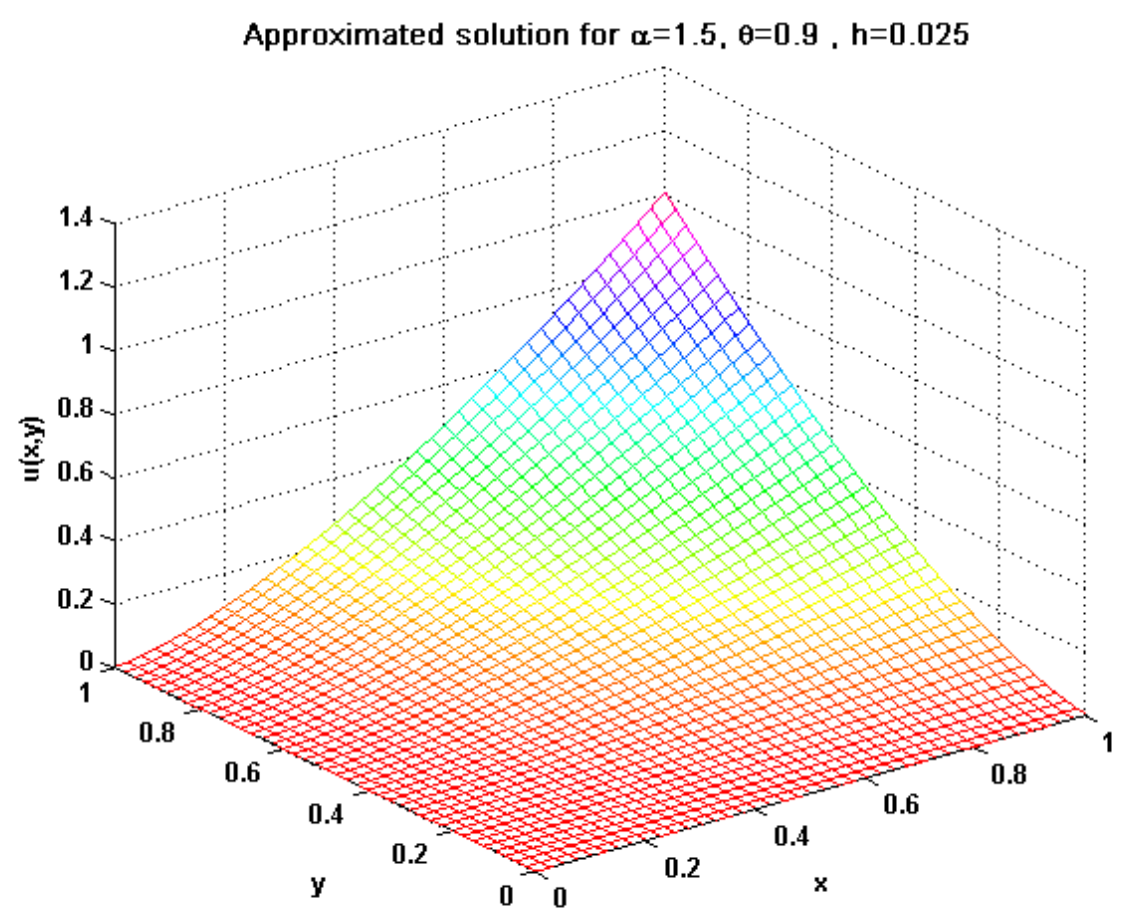

Figure 2: 3D meshed surface plot of numerical solutions for the Example 5.1 with $\alpha=1.5, \theta=0.9, h=0.025$

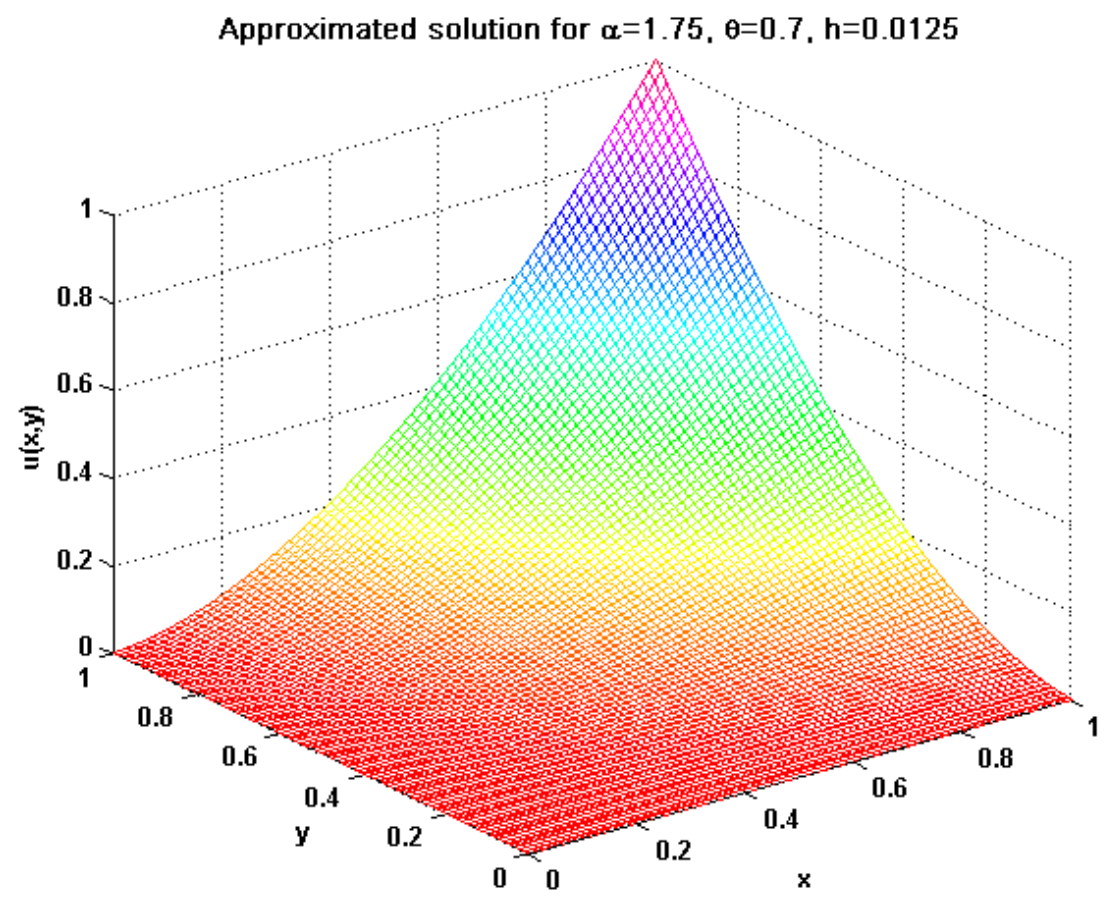

Figure 3: 3D meshed surface plot of numerical solutions for the Example 5.1 with $\alpha=1.75, \theta=0.7$, $h=0.0125$ 


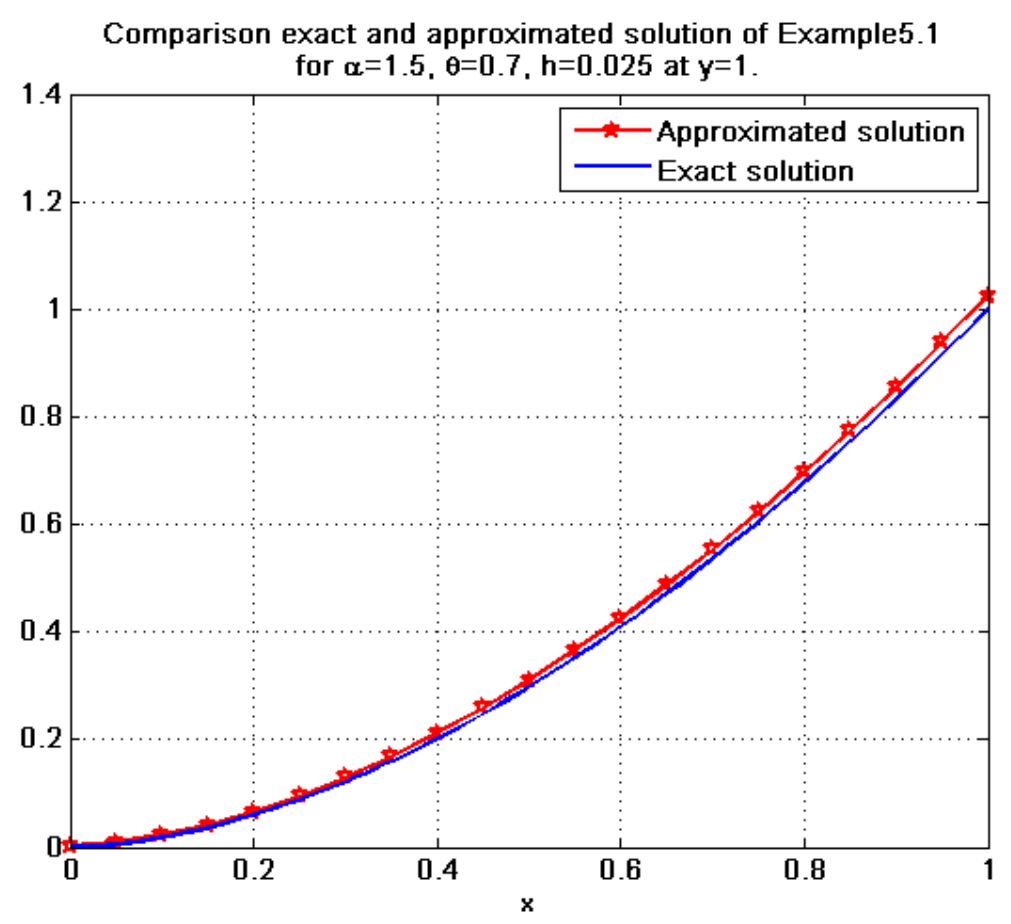

Figure 4: Comparison exact and approximated solution of Example 5.1 for $\alpha=1.5, \theta=0.7, h=0.025$ at $y=1$.

\section{Conclusion}

In this paper we presented a numerical scheme for solving fractional Poisson equation. The method employed to find the numerical solutions of these equations is based on the Grunwald estimates for Rieman-Liouvile fractional derivative. The computational results are found to be in good agreement with the exact solutions.

\section{ACKNOWLEDGEMENTS}

The authors would like to thank the referees and reviewers for their valuable comments and suggestions to improve the quality of the paper.

\section{References}

[1] M. Aslefallah, D. Rostamy. A Numerical Scheme For Solving Space-Fractional Equation By Finite Differences Theta-Method, Int. J. of Adv. in Aply. Math. and Mech., 1(4) , (2014),1-9.

[2] D.A. Benson, M.M. Meerschaert, J. Revielle. Fractional calculus in hydrologic modeling: A numerical perspective, Advances in Water Resources ,51 (2013), 479-497.

[3] A. Borhanifar, S. Valizadeh, A Fractional Finite Difference Method for Solving the Fractional Poisson Equation Based on the Shifted Grünwald Estimate, Walailak J. Sci. \&Tech.;10(5), .(2013), 427-435. 
[4] R. Gorenflo, F. Mainardi, E. Scalas, M. Raberto, Fractional calculus and continuous-time finance, III. The diffusion limit, Mathematical finance (Konstanz, 2000), Trends in Mathematics, (2001), 171-180.

[5] R. Hilfer, Applications of Fractional Calculus in Physics, World Scientific, Singapore. 2000.

[6] M. Alipour, D. Rostamy, Solving nonlinear fractional differential equations by Bernstein polynomials operational matrices, The J. of Math. And computer sci. (TJMS); 5(3), (2012).185-196.

[7] B. Lundstrom, M. Higgs, W. Spain, A. Fairhall, Fractional differentiation by neocortical pyramidal neurons, Nature Neuroscience 11, (2008). 1335-1342.

[8] M.M. Meerschaert, C. Tadjeran, Finite Difference Approximations for two-sided space-fractional partial differential equations, Applied Numerical Mathematics;56, (2006).80-90.

[9] M.M. Meerschaert, C. Tadjeran, H.P. Scheffler, A second-order accurate numerical approximation for the fractional diffusion equation, Journal of Computational Physics,213, .(2006)..205-213.

[10] M.M. Meerschaert, D.A. Benson, B. Baeumer. Multidimensional advection and fractional dispersion, Physical Review E 59, (1999). 5026-5028.

[11] E. Salehpoor, H. Jafari, Variational iteration method: A tool for solving partial differential equations, The J. of Math. And Computer Sci. (TJMS); 2(2), (2011).388-393.

[12] I. Podlubny. Fractional Differential Equations, Academic Press, San Diego.1999.

[13] J.P. Roop, Computational aspects of FEM approximation of fractional advection dispersion equations on bounded domains, Journal of Computational and Applied Mathematics 193(1),(2006).243268.

[14] Y.A. Rossikhin, M.V. Shitikova, Application of fractional derivatives to the analysis of damped vibrations of viscoelastic single mass systems, Acta Mechanica 120,(1997). 109-125.

[15] L. Sabatelli, S. Keating, J. Dudley, P. Richmond, Waiting time distributions in financial markets, European Physical Journal B 27, (2002). 273-275.

[16] S. Samko, A. Kibas, O. Marichev, Fractional Integrals and derivatives: Theory and Applications, Gordon and Breach, London.1993.

[17] R. Schumer, D.A. Benson, M.M. Meerschaert, S.W. Wheatcraft, Eulerian derivation of the fractional advection-dispersion equation, Journal of Contaminant Hydrology 48, (2001). 69-88.

[18] R. Schumer, D.A. Benson, M.M. Meerschaert, B. Baeumer, Multiscaling fractional advectiondispersion equations and their solutions, Water Resources Research 39, (2003). 1022-1032. 
[19] G.D. Smith, Numerical Solution of Partial Differential Equations : Finite Difference Methods, Oxford University Press.1978

[20] M.S. Tavazoei, M. Haeri, Describing function based methods for predicting chaos in a class of fractional order differential equations, Nonlinear Dynamics 57 (3), (2009). 363--373.

[21] S.B. Yuste, L. Acedo, K. Lindenberg, Reaction front in an $A+B \rightarrow C$ reaction-subdiffusion process, Physical Review E 69(3), (2004). 036-126.

[22] Y.W. Zhang, Formulationand solution to time-fractional Sharma-Tassoo-Olever equation via variational methods, Int. J. of Appl. Math and Mech. 9 (18): .(2013). 15-27. 